\title{
HABILIDADES ARGUMENTATIVAS Y RIQUEZA LÉXICA EN UN CURSO DE EDUCACIÓN SUPERIOR
}

\author{
ARGUMENTATIVE SKILLS AND LEXICAL \\ RICHNESS IN A HIGHER EDUCATION COURSE
}

\author{
$*$ \\ Gabriel Valdés-León \\ Universidad Católica Silva Henríquez \\ gvaldeslducsh.cl
}

En el contexto de un trabajo que se enmarca en los estudios de alfabetización académica, esta investigación se propone explorar la relación entre habilidades argumentativas y riqueza léxica en un curso de escritura para estudiantes de primer año de pedagogía. A través de análisis descriptivos de corte cuantitativo, las habilidades argumentativas fueron categorizadas en tres niveles considerando dos grandes aspectos, a saber, estructura (planteamiento de posición, fundamentación y contraargumentación) y calidad de los argumentos (aceptabilidad, relevancia y suficiencia); por su parte, abordamos la riqueza léxica mediante la identificación de la densidad léxica y del intervalo de aparición de palabras nocionales. Tanto los resultados de calidad argumentativa como los de riqueza léxica de los textos analizados se ubican, principalmente, en los niveles inicial e intermedio, lo que se condice con los trabajos que han abordado estas competencias en estudiantes de nuevo ingreso. Si bien no es posible establecer una relación de interdependencia, los resultados nos llevan concluir que la riqueza léxica es menor cuanto mayor es la calidad de los argumentos, lo que invita a indagar en los aspectos lingüístico-discursivos que inciden en ello.

Palabras clave: habilidades argumentativas, riqueza léxica, alfabetización académica, escritura en la universidad 


\section{6 - Cogency, Journal of reasoning and argumentation}

This research aims to explore the relationship between argumentative skills and lexical richness in a writing course for first-year pedagogy students. Through quantitative descriptive analysis, argumentative skills were categorized into three levels considering two major aspects, namely, structure and quality of the arguments; for its part, we address the lexical richness by identifying the lexical density and the interval of appearance of notional words. Both the argumentative quality results and the lexical richness of the analyzed texts are located mainly at the initial and intermediate levels, which is consistent with the works that have addressed these competencies in new students. Although it is not possible to establish a relationship of interdependence, the results lead us to conclude that the lexical richness is lower the higher the quality of the arguments, which invites us to investigate the linguistic-discursive aspects that affect it.

Keywords: argumentative skills, lexical richness, academic literacy, university composition 


\section{Introducción}

En el ámbito sudamericano, se han escrito muchas páginas, sobre todo en los últimos 20 años, en torno a la importancia de apoyar a los estudiantes que ingresan a la educación superior a través de medidas que van desde lo pedagógico hasta lo político-institucional (Carlino, 2003). Esta necesidad no solo se justifica por los bajos resultados de jóvenes en etapa preuniversitaria en el plano de la lectura y la escritura (Villarroel, García, Melipillán, Achondo y Sánchez, 2015), sino también por las nuevas demandas cognitivas que el ingreso a la educación terciaria representa: "El acceso a la universidad supone nuevos roles lectores, que desconocen los ingresantes provenientes de otras subculturas académicas (...). Los alumnos necesitan participar en recurrentes situaciones que andamien sus intentos inicialmente infructuosos" (Estienne y Carlino, 2004, p.7).

Aunque los trabajos orientados hacia el fortalecimiento de las competencias de lectura y escritura en la educación superior poseen una larga tradición en el ámbito anglosajón, lo que se ha publicado en español, específicamente en el contexto americano, es relativamente reciente. Navarro, Ávila, Tapia-Ladino, Cristovão, Moritz, Narváez y Bazerman (2016) realizan un panorama de los estudios sobre lectoescritura en educación superior en Latinoamérica y dan cuenta del creciente interés que este tema generó, principalmente, a partir del año 2000: de forma paulatina, se implementaron cursos curriculares y extracurriculares en las universidades de América Latina (p.e., Oyarzún y Valdés, 2019), se crearon redes de académicos interesados en este tema (p.e., REDLESS), se establecieron acuerdos de colaboración de carácter internacional (p.e., la Red Latinoamericana de Centros y Programas de Escritura) y aumentó la cantidad de publicaciones, tanto teóricas como aplicadas.

Diversas son las temáticas sobre las cuales se ha publicado en este ámbito, principalmente sobre lectura (Cisneros, Olave y Rojas, 2013; Sologuren, 2020), escritura (Lillo, Fuentes y Vega, 2020; Calle-Arango, 2020) y el valor epistémico de esta última (Arévalo, 2018; Acosta, Cubillo y Águila, 2020). En este escenario, dado el interés de nuestro trabajo, nos enfocaremos en relevar cómo se ha abordado la argumentación, por una parte, y el léxico, por otra, en estudios aplicados en la educación universitaria. Sobre el primer tema, predominan investigaciones realizadas en cursos de escritura desde una perspectiva transversal, vale decir, abordando el fenómeno de la lectoescritura como una habilidad genérica.

Algunos trabajos, como el de Bañales, Vega, Araujo, Reyna y Rodríguez (2015), Henao, Londoño-Vásquez y Frías-Cano (2017) y Valdés-León y Barrera (2020), dan cuenta de esfuerzos pedagógicos bien estructurados a través de secuencias didácticas cuya finalidad se orienta hacia el mejoramiento de competencias argumentativas utilizando géneros académicos como el resumen, la reseña crítica y el ensayo. Con el mismo nivel de especificidad, 
pero en el contexto de secuencias didácticas disciplinares que incorporan la oralidad, la escritura y el trabajo en equipo, investigaciones como las de Sánchez, González y García (2013) y Molina (2015) permiten evidenciar la importancia que se ha dado a la argumentación en la educación superior durante las últimas décadas, sobre todo al considerar el papel que esta tiene en el rendimiento académico.

Frente a estos estudios, de corte evidentemente pedagógico-didáctico, existen otros que han abordado la argumentación desde una perspectiva un tanto más especializada: a modo de ejemplo, la calidad argumentativa de textos escritos por estudiantes de educación superior ha sido estudiada en el trabajo de Larraín, Freire, Moretti, Requena y Sabat (2015), quienes analizan un corpus de 336 escritos. Para ello, elaboraron una rúbrica de evaluación que le otorga un papel secundario a otros aspectos discursivos, como la ortografía, la coherencia y la cohesión. Otro ejemplo al respecto es la investigación de León (2019), que se enfoca en la construcción de los argumentos de estudiantes de doctorado del área de las humanidades desde una mirada pragmadialéctica de la argumentación.

Dada la vasta cantidad de perspectivas que se pueden adoptar en torno al concepto de argumentación, nos parece pertinente señalar que esta investigación adhiere a la propuesta de Larraín et al. (2015), pues lo aborda en el marco de una investigación que, al igual que nuestro trabajo, se llevó a cabo en el contexto de la educación superior chilena. Así, entendemos que las habilidades argumentativas "pueden entenderse en términos de la calidad del desempeño que muestran los hablantes en la construcción y evaluación de argumentos en situaciones argumentativas" (Larraín et al., 2015, p.204). En este mismo trabajo, los autores precisan que esta calidad se evidencia si los argumentos cuentan con premisas aceptables, relevantes y suficientes para sostener una conclusión.

En el plano de la competencia léxica, el énfasis de los estudios latinoamericanos que se enmarcan en la alfabetización académica está en la relación entre esta y otras (sub)competencias comunicativas, como la lectura y la escritura (p.e., Gonzalo, 2016; Valdés-León, 2020). En este sentido, suele tener un papel más bien secundario, vista como un elemento más dentro de una serie de aspectos lingüístico-discursivos que forman parte de la calidad de un texto. No obstante, es posible encontrar algunos estudios que, sin caer en el descriptivismo extremo, dan cuenta de experiencias pedagógicas en la educación superior que ponen el foco en el componente léxico. Al respecto, podemos ejemplificar con trabajos como los de Cerda, Salcedo, Pérez y Marín (2017), Gonzalo (2018) y Cisneros y Olave (2019), quienes, aplicando metodologías cuantitativas, relevan el papel del léxico como elemento clave en procesos de enseñanza-aprendizaje en la formación universitaria.

Si bien el caudal de trabajos en torno a la escritura y la enseñanza de la argumentación escrita en el ámbito de la educación superior han ido en aumento durante los últimos años, 
la incidencia que tiene la competencia léxica en el desempeño de estas ha quedado en un segundo plano, por lo menos en el campo de estudios de español como lengua materna (Valdés-León, 2020). Esto, sin duda, representa un desafío para los investigadores y docentes interesados por la formación universitaria, sobre todo al momento de observar, por una parte, los avances que se han alcanzado al relacionar estas habilidades comunicativas en el ámbito anglosajón (p.e., Pan y Liu, 2019; Wood, Schatschneider y Hart, 2020) y, por otra, el impacto que estas poseen en el éxito académico de los universitarios (p.e., Acevedo, 2006; Gonzalo, 2016).

Considerando lo anterior, este trabajo contribuye con los estudios sobre la competencia comunicativa en distintos planos: en primer lugar, permite aportar en un ámbito muy específico en el desarrollo de estas, a saber, la perspectiva disciplinar -y no genérica ni transversal- que se les otorga en la formación de lingüistas, filólogos y profesores de lengua; luego, representa un aporte para los estudios sobre argumentación en la educación terciaria, en tanto indaga en las relaciones entre esta y la competencia léxica; por último, se suma a la acotada lista de investigaciones que, en nuestra lengua, ponen el foco sobre el componente léxico como una competencia clave en procesos de enseñanza-aprendizaje (Riffo, Reyes, Novoa, Véliz y Castro, 2014).

En ese contexto, la investigación que aquí se presenta busca contribuir con este campo de estudios poco explorado a través de un estudio que relaciona dos competencias clave para la educación terciaria: las competencias argumentativa y léxica. Para ello, este trabajo tiene como objetivo explorar la relación entre habilidades argumentativas y riqueza léxica en un curso de escritura para 25 estudiantes de primer año de pedagogía a partir del análisis de un corpus compuesto por 11 textos argumentativos, los cuales fueron elaborados en equipos de dos o tres alumnos.

\section{Materiales y métodos}

Esta sección está destinada a describir las decisiones metodológicas adoptadas en la presente investigación.

\section{Diseño y participantes}

En el marco de un diseño preexperimental, se elaboró un estudio de caso con una sola medición (Cruz, Olivares y González, 2014). De esta experiencia participaron 25 estudiantes, vale decir, la totalidad del curso Producción oral y escrita, curso correspondiente al primer semestre de la carrera de pedagogía en castellano de una universidad privada chilena. A este grupo, se le asignó una tarea de escritura argumentativa que fue desarrollada en equipos de 
$110-$

entre dos y tres estudiantes. Gracias a esto, se obtuvieron 11 textos, los que fueron sometidos a un análisis lexicométrico, por una parte, y argumentativo, por otra.

\section{Procedimiento}

Esta investigación toma los trabajos escritos por 25 estudiantes en el contexto de la evaluación final del curso Producción oral y escrita I de la carrera de Pedagogía en Castellano. Esta evaluación invitaba a los estudiantes a redactar un texto en el que debían resolver un problema de carácter pedagógico, lo que implicaba dejar en evidencia aspectos disciplinares y didácticos abordados durante el curso mediante la redacción de un texto argumentativo. Para llevar a cabo esta instancia evaluativa, se utilizó la plataforma virtual tanto para plantear la consigna como para recibir los escritos de los alumnos, y se adaptó un desafío de escritura basado en el proyecto Activando la Resolución de Problemas en las Aulas (ARPA, 2020).

Respecto de la evaluación, cabe destacar que el análisis llevado a cabo en esta investigación no incidió en la calificación de los estudiantes, pues para ello se utilizó un instrumento de evaluación mucho más coherente con los objetivos del curso. Asimismo, antes de realizar el envío de su evaluación, los estudiantes que participaron de esta experiencia entregaron por escrito, de forma voluntaria, un consentimiento en el cual se indicaba que los datos recogidos serían utilizados con fines investigativos.

\section{Variables e instrumentos}

Dos fueron las variables consideradas: habilidades argumentativas y riqueza léxica. Para la primera de estas, se adaptó la rúbrica elaborada por Larraín et al. (2015), quienes, a su vez, la diseñaron sobre la base de los trabajos de Leitão (2000) y Govier (1999). Este instrumento permite clasificar los textos en tres niveles de acuerdo con su calidad argumentativa considerando "dos aspectos de la producción argumentativa: estructura (sub-aspectos: planteamiento de posición, fundamentación y contraargumentación) y calidad (aceptabilidad, relevancia y suficiencia)" (Larraín et al., 2015, p.212). Este instrumento cuenta con un puntaje máximo de 3 pts. 


\section{Tabla 1}

Aspectos considerados en la producción argumentativa

\begin{tabular}{|c|c|c|}
\hline ASPECTOS & SUBASPECTOS & DEFINICIÓN \\
\hline \multirow{3}{*}{ Estructura } & $\begin{array}{l}\text { planteamiento o formulación de } \\
\text { una posición }\end{array}$ & se plantea claramente una posición o conclusión. \\
\hline & fundamentación & $\begin{array}{l}\text { cantidad y articulación de premisas y subpremisas formuladas } \\
\text { en apoyo a la conclusión. }\end{array}$ \\
\hline & contraargumentación & $\begin{array}{l}\text { formulación de argumento que debilita la relación entre premi- } \\
\text { sas y conclusión, y se responde a este. }\end{array}$ \\
\hline \multirow{3}{*}{ Calidad } & aceptabilidad & $\begin{array}{c}\text { grado en el que las premisas esgrimidas tanto a favor como en } \\
\text { contra de una conclusión son aceptables según el conocimiento } \\
\text { disponible del evaluador. }\end{array}$ \\
\hline & relevancia & $\begin{array}{l}\text { grado en el que las premisas aumentan la posibilidad de que la } \\
\text { conclusión sea aceptada. }\end{array}$ \\
\hline & suficiencia & $\begin{array}{l}\text { grado en el que el conjunto de premisas es suficiente para afir- } \\
\text { mar una conclusión. }\end{array}$ \\
\hline
\end{tabular}

Fuente: elaborada a partir de Larraín et al. (2015, p.212).

Por su parte, para la medición de léxico, se adoptó la propuesta de López Morales (2011), quien valora la riqueza léxica de acuerdo con dos factores: porcentaje de palabras nocionales diferentes (PV) e intervalo de aparición de palabras nocionales (IA). Las fórmulas que el autor ofrece son las siguientes (p.20):

$\mathrm{PV}=\frac{\mathrm{V}^{\star} 100}{\mathrm{~N}}$

PV: porcentaje de vocablos

V: total de palabras que poseen contenido semántico

$\mathrm{N}$ : total de vocablos del texto

$\mathrm{IA}=\underline{\mathrm{N}}$

PN

IA: Intervalo de aparición de palabras con contenido nocional

$\mathrm{N}$ : total de palabras del texto

PN: palabras nocionales 


\section{Procesamiento de los datos}

Sobre la base del aprendizaje basado en problemas, los estudiantes elaboraron un texto argumentativo para responder a una situación mediante la adopción de un rol y, de esta manera, movilizar las competencias argumentativas de manera contextualizada.

Posteriormente, se procedieron a revisar los trabajos con la rúbrica que mide la calidad argumentativa. Para ello, siguiendo a Martínez-Gamboa (2010), se promediaron los resultados de dos evaluadores expertos con el fin de disminuir los sesgos en la evaluación. Posteriormente, se categorizaron los 11 textos en tres niveles: nivel inicial ( 2 textos, entre 0 y 1 pts), nivel intermedio ( 5 textos, entre 1 y 2 puntos) y nivel avanzado ( 4 textos, entre 2 y 3 puntos). Luego, cada uno de estos subcorpus fue sometido a un análisis lexicométrico utilizando el programa Iramuteq con la finalidad de recoger los datos que nos permitiesen identificar el nivel de riqueza léxica para cada uno de ellos.

\section{Resultados}

En consonancia con lo anterior, los resultados se organizan de acuerdo con los tres niveles de desempeño argumentativo alcanzado por los estudiantes. En este sentido, daremos cuenta, en primer lugar, de los datos para el nivel inicial en el plano argumentativo y, al mismo tiempo, de las estadísticas lexicométricas para ese subcorpus. Seguidamente, realizaremos lo propio con los textos de niveles intermedio y avanzado.

\section{Textos de nivel inicial}

Como señalamos con anterioridad, solo dos textos se ubicaron en el nivel inicial. Este subcorpus se caracteriza por un muy bajo desempeño en el plano argumentativo, lo que se explica, entre otros aspectos, por la ausencia total de contraargumentos (ver Gráfico 1). Asimismo, destaca que solo el planteamiento de la postura de los hablantes frente al tema se ubica por sobre un nivel aceptable, vale decir, superior al $60 \%$.

\section{Gráfico 1.}

Resultados de las habilidades argumentativas en el subcorpus nivel inicial

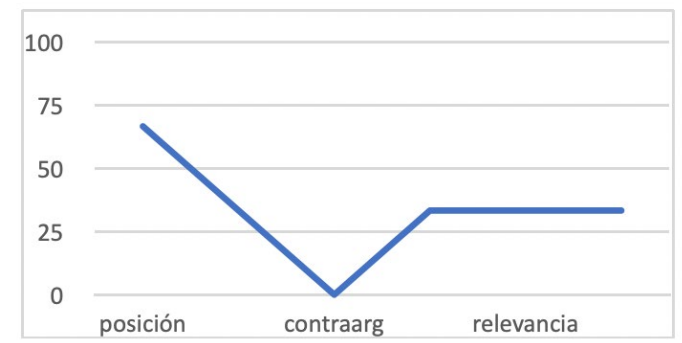


Los datos obtenidos al procesar los escritos utilizando el programa Iramuteq (ver Gráfico 2) indican que los textos que componen este subcorpus poseen 349,5 palabras en promedio. $\mathrm{Al}$ aplicar las fórmulas planteadas por López Morales (2011), tenemos que el porcentaje de vocablos (PV) se obtiene a partir de la relación entre el total de palabras con contenido semántico (o 'número de formas' en la nomenclatura de la aplicación) y el total de vocablos (u 'ocurrencias'). Al realizar dicha operación, obtenemos PV=40,6. Por su parte, el intervalo de aparición de palabras nocionales (IA) se obtiene al dividir el número total de lexías en el texto por el total de palabras nocionales presentes. En este caso, $I A=2,9$, lo que significa que hay que esperar 2,9 palabras para que aparezca una palabra con contenido nocional.

\section{Gráfico 2.}

Logaritmo de frecuencia y de rango en el subcorpus nivel inicial.

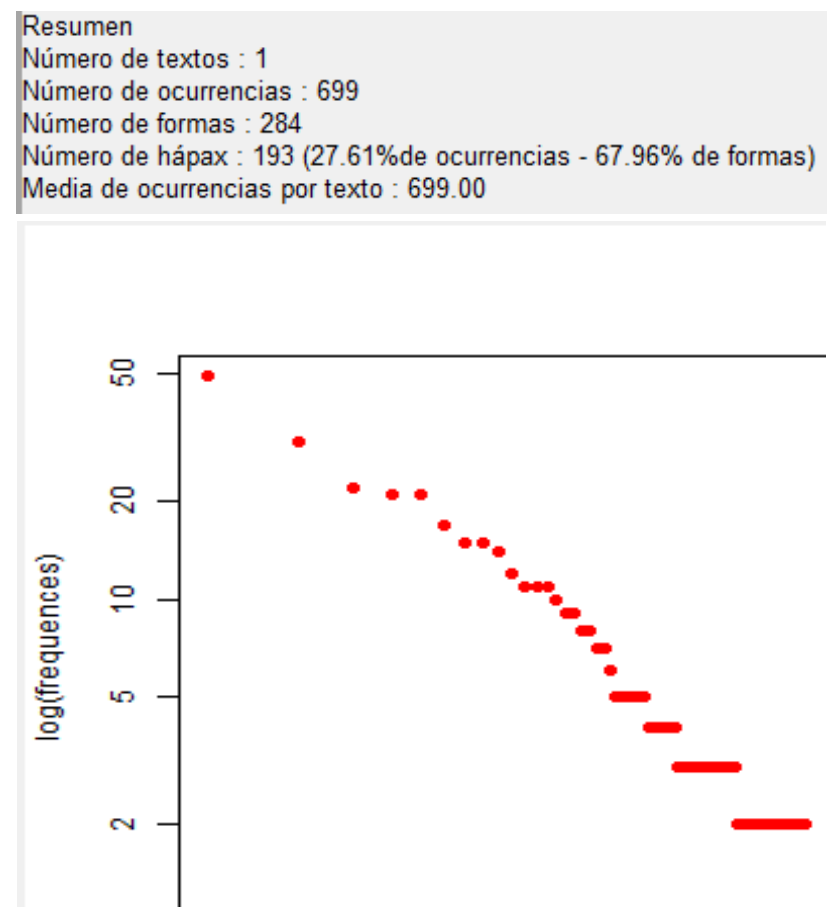

\section{Textos nivel intermedio}

$\mathrm{Al}$ observar el gráfico 3, se aprecia un aumento considerable en relación con el nivel inicial (ver Gráfico 1). Aunque baja, hay presencia de contraargumentos, y los niveles del resto de los criterios se acercan mucho más a niveles de aprobación. La presencia de una posición clara sigue siendo el aspecto más fortalecido de los textos, mientras que la presencia de contraargumentos, el que presenta menor nivel de logro. 


\section{Gráfico 3.}

Resultados de las habilidades argumentativas en el subcorpus nivel intermedio

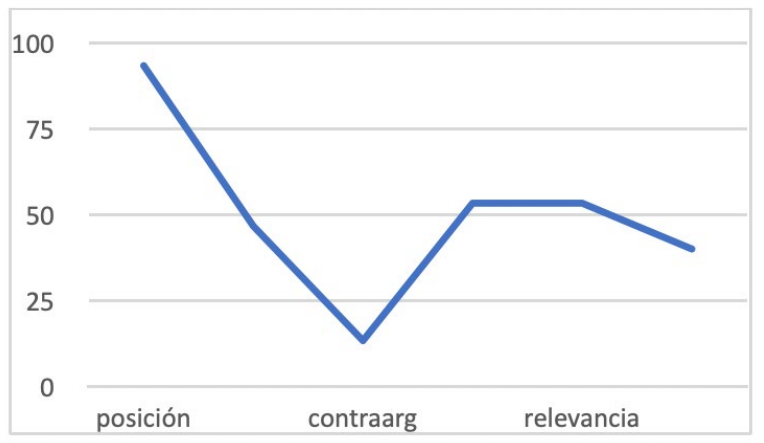

En el plano léxico, cabe señalar que, en promedio, los cinco textos que conforman este subcorpus poseen 498,2 palabras en total, es decir, casi 150 palabras más que los textos de nivel inicial. A partir de los datos que obtenemos del gráfico 4, podemos calcular valores de $P V=26,9$ e $I A=3,9$, ambos considerablemente más bajos que en el nivel inicial (-13,7\% y -1, respectivamente). Para comprender con mayor profundidad la importancia de estos números al momento de valorar la riqueza léxica, López Morales (2011, citando a Ávila, 2001) señala que, en un estudio realizado en Ciudad de México, "entre los hablantes del nivel culto, el índice general de riqueza léxica fue de 68,5". Con este dato como referencia, resulta llamativo que los textos de menor nivel argumentativo evidencian un mayor nivel de riqueza léxica.

\section{Gráfico 4.}

Logaritmo de frecuencia y de rango en el subcorpus nivel intermedio
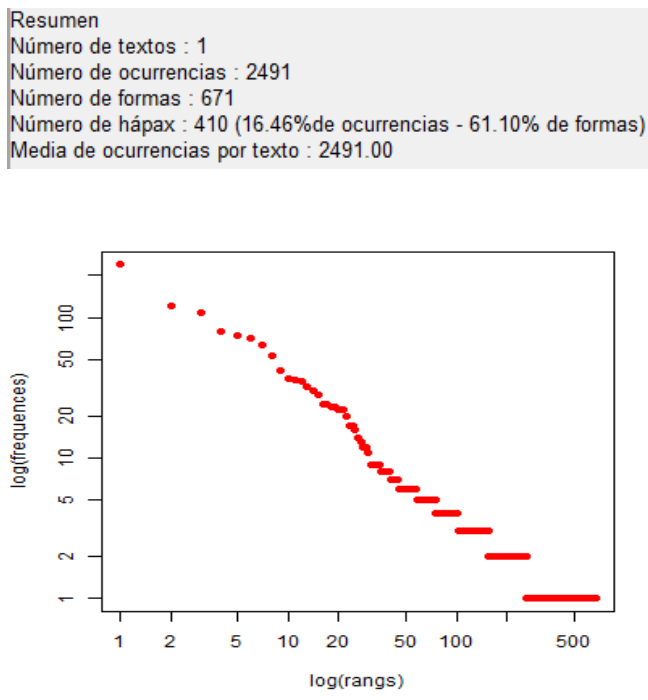


\section{Textos nivel avanzado}

El Gráfico 5 da cuenta de un desarrollo mucho más alto en los subcriterios postura, fundamentación, aceptabilidad, relevancia y suficiencia, todos por sobre un $80 \%$ de logro. La contraargumentación, por su parte, sigue siendo uno de los aspectos más débiles. Sin embargo, los resultados en este ámbito fueron mayores a $20 \%$, por tanto, aún siendo un número bajo, supera a los dos niveles anteriores. En este sentido, el contraste entre los niveles inicial, intermedio y avanzado demuestra un aumento progresivo en cada uno de los subcriterios y, en el análisis interno, la tendencia apunta hacia un alto nivel de logro en la postura que se defiende en contraste con un bajo porcentaje en el ámbito de la contraargumentación. El resto de los subcriterios suele presentarse de manera relativamente homogénea.

\section{Gráfico 5.}

Resultados de las habilidades argumentativas en el subcorpus nivel avanzado

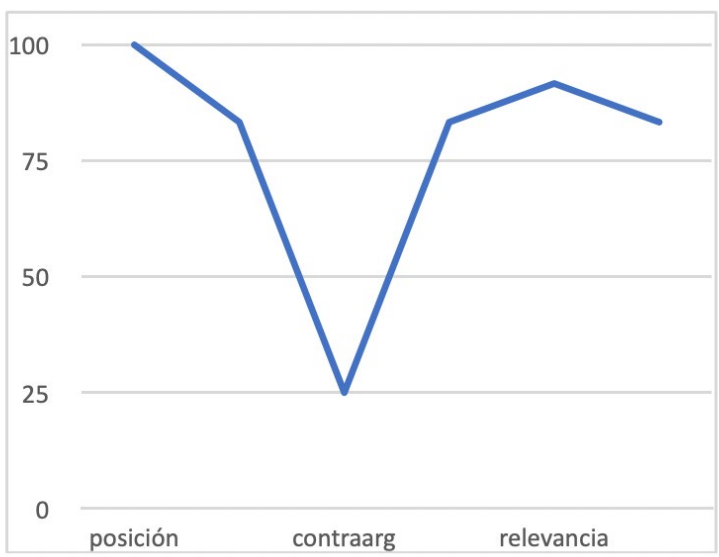

En cuanto a las estadísticas lexicométricas, el subcorpus de textos de nivel avanzado en el plano argumentativo está compuesto por cuatro escritos, cuya extensión es de 544 palabras por texto, o sea, 46 palabras más que los de nivel intermedio y 194,5 más que los de nivel inicial. Al considerar los datos expuestos en el gráfico 6, los índices $\mathrm{PV}=29,1$ e IA=3,8 resultan ser bastante cercanos a los presentados por el subcorpus de nivel intermedio $(+2,2$ y $-0,1$, respectivamente), por lo que la brecha con los textos de nivel inicial es también bastante amplia. Así, considerando el porcentaje de vocablos y el intervalo de aparición de palabras, podemos situar el índice de riqueza léxica de este subcorpus en un nivel bastante bajo. 


\section{Gráfico 6.}
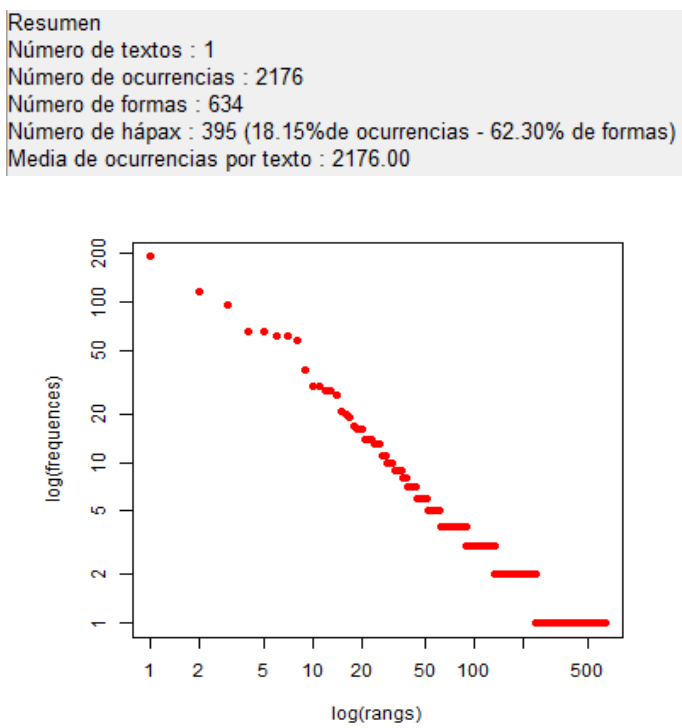

\section{Discusión}

Dejando de lado los metaanálisis y los estudios bibliométricos, las investigaciones sobre competencias comunicativas en la educación superior suelen adoptar dos grandes miradas: por una parte, una perspectiva didáctico-académica y, por otra, una de carácter profesionalizante. Respecto de la primera, que es la que nos atañe, los objetivos de los textos suelen orientarse hacia el desarrollo de habilidades comunicativas que permitan a los estudiantes desempeñarse exitosamente durante su formación universitaria.

La tendencia, en esta línea de trabajos, apunta principalmente hacia el desarrollo de competencias de lectura y escritura desde una perspectiva transversal, ya sea desde propuestas didácticas (por ejemplo, Álvarez y Taboada, 2016; Oyarzún y Valdés, 2019) hasta análisis de experiencias pedagógicas (entre ellos, Bañales et al., 2015; Torres, 2018; Chiribonga, Yumisaca y Estrada, 2018; Valdés-León, 2020). En términos generales, las investigaciones que comparten esta orientación suelen sustentarse en la importancia que poseen las competencias comunicativas, sobre todo de lectura y escritura, en el éxito académico: “... el desarrollo adecuado de [la lectura y la escritura] facilita alcanzar niveles de logro que se condicen con el éxito académico y profesional, respondiendo a la necesidad de establecer gestiones que promuevan y beneficien el aprendizaje" (Valenzuela, 2019, p.196).

Debido a que las demandas cognitivas y lingüístico-discursivas de los textos argumentativos suelen ser mayores (Estienne y Carlino, 2004), se le ha dado bastante atención a la argumentación en la universidad: en la educación terciaria, se enfatizan "los problemas de acceso de los estudiantes al conocimiento disciplinar, debido a las dificultades de comprensión y producción de los textos académicos, cuya dimensión argumentativa es 
fundamental" (Padilla, 2012, p.33). En efecto, han hecho eco de esta necesidad trabajos como los de Valdés-León y Barrera (2020), quienes proponen la reseña crítica como un género discursivo que, dada su brevedad y la simpleza de su superestructura, permiten un primer acercamiento hacia la argumentación en el mundo académico. En este sentido, el trabajo que aquí ofrecemos contribuye con las investigaciones sobre argumentación en la educación superior desde el enfoque de los estudios del léxico, tema que ha sido muy poco abordado en el ámbito de enseñanza de español como lengua materna y que, a partir de los resultados, posee un alto valor al momento de evaluar el desempeño argumentativo escrito.

Los resultados de la investigación que aquí se presenta siguen la línea del trabajo de Larraín et al. (2015), quienes se enfocan en la competencia argumentativa por sobre otros aspectos lingüístico-discursivos. Este acercamiento, de carácter mucho más especializado, permite establecer relaciones entre la calidad argumentativa y otras subcompetencias comunicativas, como la calidad textual, la comprensión lectora o, como en este caso, la competencia léxica. Si bien el objetivo de su trabajo era comparar el nivel argumentativo entre la educación universitaria y la educación técnica, los resultados que se recogen en el nivel terciario son bastante similares a los que arroja nuestra investigación: la formulación del punto de vista como el aspecto con mejor rendimiento y la baja presencia de contraargumentación en los escritos. Respecto de este último elemento, sin duda el más llamativo, trabajos como Hódar, Pérez-Martínez, Morales, Martín-Cuenca y Iáñez-Pareja (2016) y Malouff, Rooke y Schutte (2018) dan cuenta de las dificultades que los alumnos ingresantes evidencian en el desempeño de la competencia discursiva, competencia que se relaciona con el carácter dialógico que posee la argumentación.

Ahora bien, en cuanto a la relación entre la riqueza léxica y las habilidades argumentativas, poco se ha escrito en el ámbito del español como lengua materna con este nivel de especificidad y desde perspectivas cuantitativas. Sí han atendido a esta relación investigaciones como las de Padilla (2012), Álvarez (2017) y Valdés-León y Barrera (2020), pero se suele atribuir un rol secundario al componente léxico, a la par de otros aspectos textuales como coherencia, cohesión y ortografía. En este sentido, nos parece necesario destacar la relación inversa que nuestros resultados permiten vislumbrar: en la medida en que aumenta la calidad argumentativa, disminuye la riqueza léxica de los textos.

Si bien los resultados de nuestra experiencia son representativos solo del acotado corpus utilizado, resulta al menos llamativo que estos vayan en dirección contraria a lo que podría esperarse, es decir, que aumentara la riqueza léxica en la medida en que aumentara también la calidad argumentativa. Así, en el afán de buscar caminos que nos permitan explicar la relación inversa que se halló, podemos recurrir a la investigación de Lillo-Fuentes y Venegas (2020), quienes relacionan la calidad de la escritura con rasgos lingüístico-discursivos 
en textos de educación superior y, entre otros aspectos, identifican que a) los textos con mayor cantidad de palabras suelen tener mayor calidad textual y b) mientras mayor es la calidad, mayor es la cantidad de mecanismos de cohesión y, consecuentemente, el uso de pronombres. Sobre esta base, el subcorpus de nivel inicial, vale decir, aquel que posee textos con menor calidad argumentativa, es aquel que contiene el menor promedio de palabras; contrariamente, el subcorpus de nivel avanzado en cuanto a habilidades argumentativas presenta los textos más extensos: promedia 544 palabras por escrito.

Escapa del alcance de este trabajo indagar en la relación que existe entre calidad textual, en un sentido amplio, y calidad argumentativa. Sin embargo, los resultados de esta experiencia, que demuestran una relación inversa entre riqueza léxica y calidad argumentativa, pueden explicarse debido a que aquellos textos que presentan una construcción argumentativa más sólida son más extensos y poseen mayor presencia de conectores, pronombres, preposiciones, etc. (es decir, un mayor número de palabras sin contenido semántico nocional, lo que se traduce en un índice bajo de riqueza léxica), rasgos que, a la luz de los resultados de Lillo-Fuentes y Venegas (2020) parecen coincidir también con textos de mejor calidad textual.

Sin afán de generalizar, nos parece que la relación que esta investigación esboza puede contribuir con la enseñanza de la argumentación en la universidad principalmente en dos grandes momentos: durante la retroalimentación y durante la evaluación. Respecto de la retroalimentación, se releva la importancia de fortalecer el adecuado uso de conectores y marcadores discursivos, entendiendo su importancia en la construcción lógica del texto y la relación que existe entre calidad argumentativa y léxico no nocional, sobre todo si consideramos que “... la fuerza contrastiva entre argumentos y contraargumentos puede manifestarse a través de las palabras utilizadas, junto con conectores adversativos o no" (Cuenca, 1995, p.29). En el plano de la evaluación, si futuros trabajos logran confirmar los hallazgos que acá presentamos, vale decir, la relación inversa entre calidad argumentativa y riqueza léxica, cabe preguntarse si mediciones sobre promedio de palabras, riqueza léxica o léxico nocional/no nocional podrían ser utilizadas como indicador de la calidad argumentativa, por ejemplo, en procesos de evaluación automatizados.

\section{Conclusiones}

Sobre la base de los resultados obtenidos, es posible concluir que, en el plano argumentativo, el mayor porcentaje de los textos se encuentra en los niveles inicial e intermedio, lo que, sin duda, es esperable para alumnos que acaban de ingresar a la educación superior. En este sentido, el análisis realizado permite relevar la necesidad de fortalecer la competencia discursiva, enfatizando el carácter dialógico de la argumentación, con el fin de fortalecer el uso de contraargumentos como elemento clave de un buen discurso argumentativo. 
En cuanto a la relación entre calidad argumentativa y riqueza léxica, concluimos que el corpus analizado evidencia una relación inversa entre ambas subcompetencias, vale decir, a mayor calidad argumentativa, menor riqueza léxica. No obstante lo acotada de esta experiencia, consideramos que estos hallazgos resultan interesantes en cuanto invitan a indagar en el papel que juega el léxico sin contenido semántico nocional en la construcción de discursos argumentativos, por una parte, y la relación que existe entre calidad textual, competencia léxica y calidad argumentativa, por otra.

Finalmente, consideramos que esta investigación propicia la reflexión respecto del valor que se les otorga a los aspectos léxicos, principalmente al léxico no nocional, en la enseñanza de la argumentación en la universidad, sobre todo al considerar el impacto que este tiene en la construcción lógica de los argumentos y al potencial que puede llegar a tener al momento de evaluarla.

\section{Referencias bibliográficas}

Acevedo, D. (2006). Estructura y patrones léxicos en informes escritos de estudiantes universitarios. Onomázein, (13), 55-71.

Acosta, I., Cubillas, F. y Águila, M. (2020). Producción de textos escritos. Lo epistémico y lo desarrollador. Ciencia e Interculturalidad, 27(02), 30-41.

Álvarez, F. (2017) Enunciación, argumentación y subjetivemos en la reformulación del discurso académico de estudiantes ingresantes a la universidad. Difusiones 1(5), 1-28

Álvarez, G., y Taboada, M. (2016). Propuestas didácticas mediadas por tecnologías digitales para el desarrollo de competencias de lectura y escritura académicas. Revista Guillermo De Ockham, 14(2), 83-91. https://doi.org/10.21500/22563202.2336

Arévalo, J. (2018). El proceso de alfabetización académica en postgrado. de la función epistémica a la difusión del conocimiento. Espirales Revista Multidisciplinaria de investigación, 2(22).

ARPA escritura. (2020). Recuperado 13 de julio de 2020, de ARPA website: https://arpa.uchile.cl/arpa-escritura/

Ávila, R. (2001). Los medios de comunicación masiva y el español internacional. II Congreso Internacional de la Lengua Española. Madrid: Instituto Cervantes. 
Bañales Faz, G., Vega López, N., Araujo Alvineda, N., Reyna Valladares, A. y Rodríguez, B. (2015). La enseñanza de la argumentación escrita en la universidad: Una experiencia de intervención con estudiantes de Lingüística aplicada. Revista mexicana de investigación educativa, 20(66), 879-910.

Calle-Arango, L. (2020). Centros y programas de escritura en las IES colombianas. Magis, Revista Internacional de Investigación en Educación, 12(25), 77-92.

Carlino, P. (2003). Alfabetización académica: un cambio necesario, algunas alternativas posibles. Educere, 6(20), 409-420.

Cerda, A., Salcedo, A., Pérez, C. y Marín, V. (2017). Futuros Profesores de Matemáticas: Rol de la Disponibilidad Léxica, Esquemas de Razonamiento Formal en Logros Académicos Durante su Formación Inicial. Formación universitaria, 10(1), 33-46. https://dx.doi.org/10.4067/S0718-50062017000100005

Chiriboga, A, Yumisaca Y. y Estrada, J. (2018). Análisis de pertinencia de las competencias para la escritura académica con los estudiantes de las carreras de ciencias experimentales. Revista Boletín Redipe, 7(8), 113-128.

Cisneros, M., Olave, G. y Rojas, I. (2013). Alfabetización académica y lectura inferencial. Bogotá: Ecoe.

Cisneros, M., y Olave, G. (2019). El léxico disciplinar en la educación universitaria. Panorama Económico, 27(1), 249-266.

Cruz, C., Olivares, S. y González, M. (2104) Metodología de la investigación. Grupo Editorial Patria.

Cuenca, M. J. (1995). Mecanismos lingüísticos y discursivos de la argumentación. Comunicación, lenguaje y educación, 7(2), 23-40.

Estienne, V. y Carlino, P. (2004). Leer en la universidad: enseñar y aprender una cultura nueva. Uni-Pluri/Versidad, 4(3), 9-17

Gonzalo, M. (2016). Evaluación de desempeño académico: La competencia léxica p r o ductiva como competencia con validez predictiva. Revista Ensayos Pedagógicos, 11(2), 159-171. 
Gonzalo, M. (2018). Adquisición de vocabulario productivo en contextos académicos superiores: análisis de su evolución a partir de un estudio longitudinal. Ensayos Pedagógicos, 13(2), 119-136.

Govier, T. (1999). The Philosophy of Argument. Newport News, Va.: Vale Press

Henao, J., Londoño-Vásquez, D. y Frías-Cano, L. (2017). Leer y argumentar en un curso de lenguaje para estudiantes de primer año de Derecho de la Institución Universitaria de Envigado (Colombia). Revista Virtual Universidad Católica del Norte, 50, 162-182.

Hódar, J. A., Pérez-Martínez, C., Morales, A. E., Martín-Cuenca, E., y Iáñez-Pareja, E. (2016). Correct your own exam. Exercises for university students to develop writing skills in biology. En SHS Web of Conferences (Vol. 26, p. 01079). EDP Sciences.

Larraín, A., Freire, P., Moretti, R., Requena, M., y Sabat, B. (2015). ¿La Universidad de Chile promueve las habilidades de argumentación escrita?: Un estudio exploratorio comparativo de estudiantes de educación universitaria y educación técnica. Calidad en la educación, (43), 201-228. https://dx.doi.org/10.4067/S0718-45652015000200007

Leitão, S. (2000a). The potential of argument of knowledge building. Human Development, 43(6), 332-360. http://dx.doi.org/10.1159/000022695

Lillo-Fuentes, F. y Venegas, R. (2020). Relación entre calidad de escritura y rasgoslingüístico-discursivos en las introducciones de los trabajos finales de grado de ingeniería civil informática. Linguamática 12(1), 3-13

López Morales, H. (2011). Los índices de riqueza léxica y la enseñanza de lenguas. $n$ Del texto a la lengua: La aplicación de los textos a la enseñanza-aprendizaje del español L2LE (pp. 15-28). Asociación para la Enseñanza del Español como Lengua Extranjera.

León, L. (2019). Habilidades de argumentación escrita en la universidad. Estudios $\lambda$ ambda. Teoría Y práctica De La didáctica En Lengua Y Literatura, 5(1), 1-31. https:// doi.org/10.36799/el.v5i1.102

Malouff, J., Rooke, S., y Schutte, N., (2018). Simple Strategies Academics Can Use to Help Students Improve Their Writing Skills Online Submission. ERIC Doc. No. ED586961 
Martínez-Gamboa, R. (2010). Evaluar la escritura en Chile: la dimensión de escritura del Sistema de Medición de la Calidad de la Educación Chilena (Simce) desde los modelos cognitivos de la producción escrita. Tesis de Magíster en Estudios Cognitivos. Santiago, Chile: Universidad de Chile.

Molina, M. E. (2015). Argumentación, escritura y prácticas de enseñanza en el ingreso a la universidad: Estudio de casos en Letras y Biología. Montevideo: Ediciones Universitarias, Unidad de Comunicación de la Universidad de la República (ucur).

Navarro, F., Ávila Reyes, N., Tapia-Ladino, M., Cristovão, V. L., Moritz, M. E. W., Narváez Cardona, E., \& Bazerman, C. (2016). Panorama histórico y contrastivo de los estudios sobre lectura y escritura en educación superior publicados en América Latina. Revista signos, 49, 78-99.

Oyarzún, R., y Valdés, G. (2019). Propuesta didáctica para el desarrollo de habilidades comunicativas en el contexto universitario: un acercamiento a la cultura discursiva en la educación superior. Aularia: Revista Digital de Comunicación, 8(1), 1-8.

Padilla, C. (2012). Escritura y argumentación académica: trayectorias estudiantiles, factores docentes y contextuales. Magis 5(10), 31-57

Pan, F., \& Liu, C. (2019). Comparing L1-L2 differences in lexical bundles in student and expert writing. Southern African Linguistics and Applied Language Studies, 37(2), 142-157.

Riffo, B., Reyes, F., Novoa, A., Véliz y Castro, G. (2014). Competencia léxica, comprensión lectora y rendimiento académico en estudiantes de enseñanza media. Literatura y lingüística, (30), 136-165.

Sánchez, L., González, J. y García, Á. (2013). La argumentación en la enseñanza de las ciencias. Revista Latinoamericana de Estudios Educativos 1(9), 11-28.

Sologuren, E. (2020). Comprender textos escritos ¿una competencia aislada? El resumen y la alfabetización académica: un acercamiento preliminar a la conexión comprensión de textos-producción textual. Tonos digital: Revista de estudios filológicos, (39), 1-28.

Torres, A. (2018). ¿Escritura disciplinar? Una experiencia a partir de la lectura crítica y la habilidad argumentativa en la universidad. Revista mexicana de investigación educativa, 23(76), 95-124. 
Valdés-León, G. (2020). Competencia léxica y escritura académica: analíticas de aprendizaje en un curso de escritura universitaria. Texto Livre: Linguagem e Tecnología. 14(1), 1-17.

Valdés-León y Barrera, L. (2020) La reseña como puerta de entrada a los géneros académicos: un estudio de caso. Revista Formación Universitaria, 13(4), 119-128.

Valenzuela Muñoz, A. (2019). Lucía Natale (coordinadora). En carrera: escritura y lectura de textos académicos y profesionales. Buenos Aires: Universidad Nacional General Sarmiento, 2012, 240 páginas. ISBN: 978-987-630-119-0. Logos: Revista de Lingüística, Filosofía y Literatura, 29(1), 196-199. DOI: 10.15443/RL2915

Villarroel Henríquez, V., García Gómez, C., Melipillán Araneda, R., Achondo Mandiola, E., y Sánchez Oñate, A. (2015). Aprender del error es un acierto: Las dificultades que enfrentan los estudiantes chilenos en la Prueba PISA. Estudios pedagógicos (Valdivia), 41(1), 293-310.

Wood, C. L., Schatschneider, C., \& Hart, S. (2020). Average One Year Change in Lexical Measures of Written Narratives for School Age Students. Reading \& Writing Quarterly, 36(3), 260-277. 